\title{
Trivial Meet and Join within the Lattice of Monotone Triangles
}

\author{
John Engbers \\ Department of Mathematics, Statistics and Computer Science \\ Marquette University \\ Milwaukee, WI, U.S.A. \\ john.engbers@marquette.edu \\ Adam Hammett \\ Department of Mathematical Sciences \\ Bethel College \\ Mishawaka, IN, U.S.A. \\ adam.hammett@bethelcollege.edu
}

Submitted: Jan 22, 2014; Accepted: Jul 10, 2014; Published: Jul 21, 2014

Mathematics Subject Classifications: 05A05, 05A16, 06A20

\begin{abstract}
The lattice of monotone triangles $\left(\mathfrak{M}_{n}, \leqslant\right)$ ordered by entry-wise comparisons is studied. Let $\tau_{\min }$ denote the unique minimal element in this lattice, and $\tau_{\max }$ the unique maximum. The number of $r$-tuples of monotone triangles $\left(\tau_{1}, \ldots, \tau_{r}\right)$ with minimal infimum $\tau_{\min }$ (maximal supremum $\tau_{\max }$, resp.) is shown to asymptotically approach $r\left|\mathfrak{M}_{n}\right|^{r-1}$ as $n \rightarrow \infty$. Thus, with high probability this event implies that one of the $\tau_{i}$ is $\tau_{\min }$ ( $\tau_{\max }$, resp.). Higher-order error terms are also discussed.
\end{abstract}

Keywords: monotone triangles; permutations; square ice; alternating sign matrices; meet; join

\section{Introduction and statement of results}

Let $n \geqslant 1$ be an integer and $[n]:=\{1,2, \ldots, n\}$. A monotone triangle of size $n$ (or Gog triangle in the terminology of Doron Zeilberger [18]) is a triangular arrangement of $n(n+1) / 2$ integers with $i$ elements in row $i(i \in[n])$ taken from the set $[n]$ so that if $a(i, j)$ denotes the $j$ th entry in row $i$ (counted from the top), then

1. $a(i, j)<a(i, j+1), 1 \leqslant j<i$, and

2. $a(i, j) \leqslant a(i-1, j) \leqslant a(i, j+1), 1 \leqslant j<i$. 
Note that this definition forces the last row to be $a(n, 1)=1, a(n, 2)=2, \ldots, a(n, n)=n$. We let $\mathfrak{M}_{n}$ denote the set of all monotone triangles of size $n$, and we let $\tau=(\tau(i, j))$ be a generic element from this set. It is well-known that there is a bijection between $\mathfrak{M}_{n}$ and the set of all $n \times n$ alternating-sign matrices (ASMs), which are the $n \times n$ matrices of $0 \mathrm{~s}, 1 \mathrm{~s}$, and $-1 \mathrm{~s}$ so that the sum of the entries in each row and column is 1 and the non-zero entries in each row and column alternate in sign. This bijection is often seen via the column-sum matrix form of an ASM; an example of a monotone triangle of size 4, along with the corresponding column-sum matrix and ASM, is given in Figure 1.

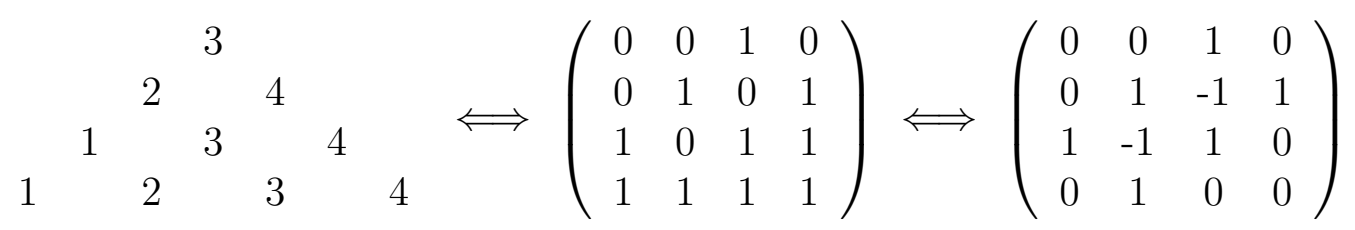

Figure 1: A monotone triangle of size 4, along with the corresponding column-sum and alternating-sign matrices.

The set $\mathfrak{M}_{n}$ is also in bijection with the square ice configurations (or six-vertex configurations) of order $n$ in statistical mechanics; for descriptions of these bijections, along with other sets in bijection with $\mathfrak{M}_{n}$, see the surveys of James Propp [15], David Bressoud and Propp [3], or the wonderful exposition by Bressoud about the proof of the ASM conjecture [2], which concerns the number of monotone triangles, denoted $A(n):=\left|\mathfrak{M}_{n}\right|$ (as this was first framed for ASMs). Specifically, the ASM conjecture is simply that

$$
A(n)=\prod_{k=0}^{n-1} \frac{(3 k+1) !}{(n+k) !}
$$

The formula (1) was first proved by Zeilberger [18], with another proof (using square ice) given by Greg Kuperberg [12]. Bressoud's book [2] not only lays out this proof in complete detail, but also delivers the historical narrative surrounding this triumph.

For the asymptotic value of $A(n)$, Bleher and Fokin [1] found that

$$
A(n)=c\left(\frac{3 \sqrt{3}}{4}\right)^{n^{2}} \times n^{-5 / 36} \times\left(1-\frac{115}{15552 n^{2}}+O\left(n^{-3}\right)\right)
$$

where $c>0$ is a constant. Using Stirling's approximation $n ! \sim e^{n \log n-n} \sqrt{2 \pi n}$ it becomes clear that $A(n)$ and $n$ ! are far apart for large $n$. We shall not need this in our present study, however. We simply need the fact that $A(n) \geqslant n$ !, which we have already seen is clear from permutation matrices being a subset of the ASMs, and can also be seen directly from monotone triangles (by iteratively building a monotone triangle from the top to the bottom, and for each new row adding one new number to the previous row). Before departing from the question of enumerating objects related to monotone triangles, 
we also mention some recent work of Ilse Fischer $[7,8,9]$ which has focused on finer enumerative questions regarding a looser definition of monotone triangles where the bottom row is simply required to be a strictly increasing collection of $n$ positive integers (rather than the specific integers $1,2, \ldots, n)$, and also the enumeration of so-called monotone trapezoids. Also, Jessica Striker [17] has recently settled the long-sought question of a bijection between descending plane partitions and ASMs in the case where the ASM is simply a permutation matrix. Striker's bijection involves a particular weighting of permutation inversions, a concept that we explore further in a forthcoming work extending this present study.

Our present work will focus exclusively on the lattice properties of $\left(\mathfrak{M}_{n}, \leqslant\right)$, where " $\leqslant$ " in this poset is defined by entry-wise comparisons. That is, given monotone triangles $\tau_{1}=\left(\tau_{1}(i, j)\right)$ and $\tau_{2}=\left(\tau_{2}(i, j)\right)$ in $\mathfrak{M}_{n}$, we define $\tau_{1} \leqslant \tau_{2}$ if and only if $\tau_{1}(i, j) \leqslant \tau_{2}(i, j)$ for $1 \leqslant j \leqslant i \leqslant n$. Of course, it is entirely possible that two monotone triangles may not be comparable at all. For example, in the case $n=3$ the following two monotone triangles are incomparable:

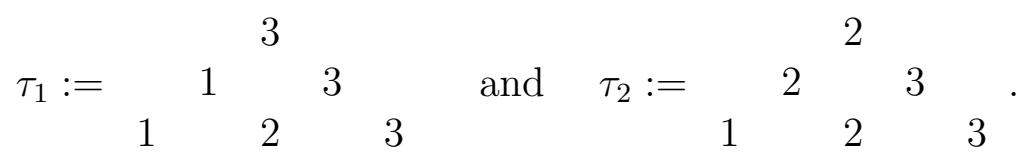

Indeed, all entries in both monotone triangles are the same except for the first entries in rows 1 and 2 , and there we have $\tau_{1}(1,1)>\tau_{2}(1,1)$ and yet $\tau_{1}(2,1)<\tau_{2}(2,1)$. However, this poset is indeed a lattice under the infimum and supremum operations

$$
\inf \left(\tau_{1}, \tau_{2}\right):=\left(\min \left\{\tau_{1}(i, j), \tau_{2}(i, j)\right\}\right) \text { and } \sup \left(\tau_{1}, \tau_{2}\right):=\left(\max \left\{\tau_{1}(i, j), \tau_{2}(i, j)\right\}\right) .
$$

Infimums and supremums for $r$ monotone triangles, where $r>2$, are defined analogously. It is left to the interested reader to check that these inf and sup operations do deliver monotone triangles, and satisfy the requirements of an infimum or supremum.

Interestingly, if we restrict this ordering to the collection of order- $n$ permutations $\mathfrak{S}_{n} \subseteq \mathfrak{M}_{n}$ with each permutation identified with its corresponding monotone triangle (through the correspondence described above), we obtain a poset $\left(\mathfrak{S}_{n}, \leqslant\right)$ referred to as the Bruhat ordering on $\mathfrak{S}_{n}$ (see, e.g., H. and Boris Pittel's work [11] or [10] for an exposition on the comparability properties of $\left.\left(\mathfrak{S}_{n}, \leqslant\right)\right)$. It was C. Ehresmann [6] who first discovered this connection between $\mathfrak{S}_{n}$ under Bruhat ordering and their monotone triangle counterparts under entry-wise comparisons. It is worth mentioning here that the infimum of two elements of $\mathfrak{S}_{n}$, when viewed as elements of $\mathfrak{M}_{n}$, need not lie in $\mathfrak{S}_{n}$; indeed, consider the $n=3$ example above in (2). There we have

$$
\inf \left(\tau_{1}, \tau_{2}\right)=\begin{array}{llllll}
2 & 1 & & 3 & \\
1 & & 2 & & 3
\end{array}
$$

and it is easy to see that this is not a monotone triangle arising from any permutation in $\mathfrak{S}_{3}$ even though both $\tau_{1}$ and $\tau_{2}$ do (they correspond to the permutations 312 and 231, 
respectively; a monotone triangle coming from a permutation will necessarily have the property that each row is a subset of the very next row). Moreover, when the permutations 312 and 231 are viewed as elements of the Bruhat poset $\left(\mathfrak{S}_{3}, \leqslant\right)$ there are two potential infimums, namely 132 and 213. However, these two permutations are incomparable in this poset and thus $\left(\mathfrak{S}_{3}, \leqslant\right)$ is not a lattice. This is a general feature of the Bruhat permutation-poset embedded within the monotone triangle lattice: the lattice property enjoyed by $\left(\mathfrak{M}_{n}, \leqslant\right)$ is not inherited by $\left(\mathfrak{S}_{n}, \leqslant\right)$. In fact, Richard Stanley notes in exercise 7.103 of $[16]$ that $\left(\mathfrak{M}_{n}, \leqslant\right)$ is the unique MacNeille completion of $\left(\mathfrak{S}_{n}, \leqslant\right)$ to a lattice. A. Lascoux and M. P. Schützenberger [13] were the first to prove this fact. We now focus exclusively on a particular feature of this lattice.

Let $\tau_{\min }$ denote the (unique) minimal element in the monotone triangle lattice. That is,

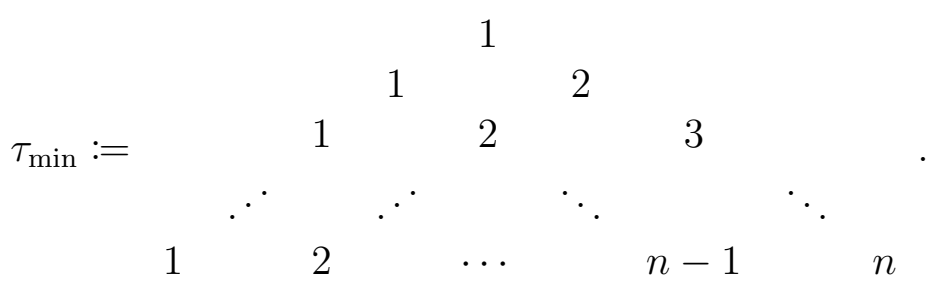

Similarly, let $\tau_{\max }$ denote the (unique) maximal element:

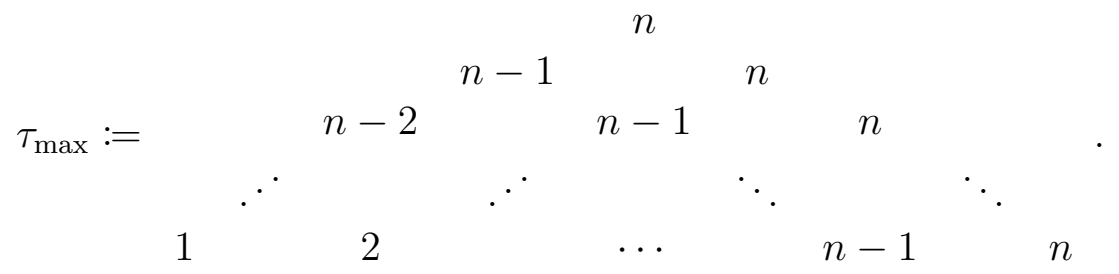

In this paper, we are interested in two questions. How likely is it that $r$ independent and uniformly random monotone triangles $\tau_{1}, \ldots, \tau_{r} \in \mathfrak{M}_{n}$ will have trivial meet, i.e. $\inf \left(\tau_{1}, \ldots, \tau_{r}\right)=\tau_{\text {min }}$, and how likely are they to have trivial join, i.e. $\sup \left(\tau_{1}, \ldots, \tau_{r}\right)=$ $\tau_{\max }$ ? Namely, we want sharp estimates on the probabilities

$$
\begin{aligned}
& \text { 1. } p_{\min }:=P\left(\inf \left(\tau_{1}, \ldots, \tau_{r}\right)=\tau_{\min }\right) \text {, and } \\
& \text { 2. } p_{\max }:=P\left(\sup \left(\tau_{1}, \ldots, \tau_{r}\right)=\tau_{\max }\right) .
\end{aligned}
$$

These questions have previously been studied for the lattice of set partitions ordered by refinement (see Canfield [4] and Pittel [14]), the lattice of set partitions of type $B$ (see Chen and Wang [5]), and the lattice of permutations under weak ordering (see H. [10]).

First of all, we show that

$$
p_{\min }=p_{\max },
$$

reducing our problem to the consideration of $p_{\min }$ only.

Proof of (3). Given a monotone triangle $\tau=(\tau(i, j)) \in \mathfrak{M}_{n}$, introduce the rank-reversed monotone triangle $\bar{\tau} \in \mathfrak{M}_{n}$ obtained by the transformation of entries

$$
\tau(i, j) \mapsto n-\tau(i, j)+1
$$


and then reversing the order of each row. For example $(n=4)$

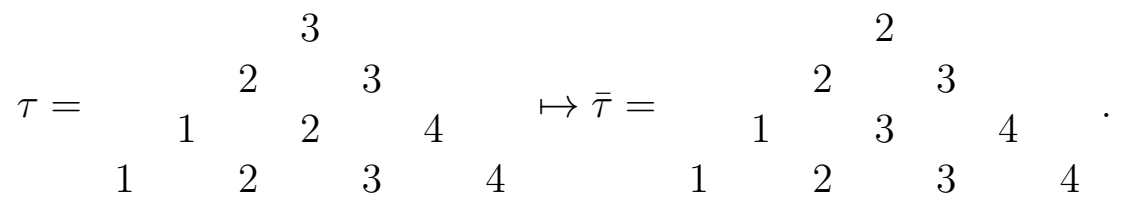

Certainly if $\tau_{1}, \ldots, \tau_{r} \in \mathfrak{M}_{n}$ are uniformly random and independent, the same is true of $\overline{\tau_{1}}, \ldots, \overline{\tau_{r}} \in \mathfrak{M}_{n}$. Moreover, it is easy to check that

$$
\inf \left(\tau_{1}, \ldots, \tau_{r}\right)=\tau_{\min } \quad \Longleftrightarrow \quad \sup \left(\bar{\tau}_{1}, \ldots, \bar{\tau}_{r}\right)=\tau_{\max } .
$$

But this means that $p_{\min }=p_{\max }$.

We are ready to state our main result.

Theorem 1. Fix an integer $r \geqslant 1$. Given $r$ independent and uniformly random monotone triangles $\tau_{1}, \ldots, \tau_{r} \in \mathfrak{M}_{n}$, we have

$$
p_{\min } \sim \frac{r}{A(n)}, n \rightarrow \infty
$$

Notice that the theorem is obvious when $r=1$, since in this case $p_{\min }=1 / A(n)$. Because of this, we assume that $r \geqslant 2$ for the remainder of the paper; this theorem says that essentially nothing changes for these $r$ : almost all ways of obtaining $\tau_{\min }$ occur when some $\tau_{i}=\tau_{\min }$ !

It is possible to extend the proof of Theorem 1 to higher-order terms. In particular, we will describe the modifications needed to obtain the following refinement.

Theorem 2. Fix an integer $r \geqslant 1$. Given $r$ independent and uniformly random monotone triangles $\tau_{1}, \ldots, \tau_{r} \in \mathfrak{M}_{n}$, we have

$$
p_{\min }=\frac{r A(n)^{r-1}+2 r(r-1) A(n-1) A(n)^{r-2}+\Theta\left(A(n-2) A(n)^{r-2}\right)}{A(n)^{r}} .
$$

Theorem 1 is a simple corollary of Theorem $2($ as $A(n-1)=o(A(n))$ and $A(n-2)=$ $o(A(n-1))$ from Lemma 4$)$, but we leave them in the paper as separate results. This is because the fundamental insights that give rise to Theorem 1 are obfuscated when refined for the proof of Theorem 2. Also, the careful reader will notice that the proof of Theorem 2 could in theory be extended to deliver further higher-order terms. However, the argument becomes more complex and involves a much finer case-by-case treatment. We hope that perhaps there is another method of proof that quickly gives all terms of $p_{\text {min }}$. In particular, other proofs of similar statements (e.g. those in $\left.[4,10,14]\right)$ proceed via generating functions, and it would be nice to find a different proof along these lines.

The remainder of the paper is laid out as follows. In Section 2, we first prove some introductory lemmas about the values $A(n)$ and then use these lemmas to prove Theorem 1. Section 3 describes how to modify this proof to obtain Theorem 2. 


\section{Proof of Theorem 1}

\subsection{Lemmas}

Before starting the proof of Theorem 1, we begin with several lemmas that help us understand how the values $A(n)$ relate to one another. Throughout we use the convention that $A(0)=1$.

Lemma 3. Let $i_{1} \geqslant i_{2} \geqslant 1$. Then $A\left(i_{1}+1\right) A\left(i_{2}-1\right) \geqslant A\left(i_{1}\right) A\left(i_{2}\right)$.

Proof. From (1) we have $A(n) / A(n-1)=\frac{(3 n-2) !(n-1) !}{(2 n-1) !(2 n-2) !}$. As this is an increasing function of $n$ for $n \geqslant 1$, the lemma follows; we leave the details to the reader.

Lemma 4. For all $n \geqslant 1$ and any constant $c \in[n]$, we have

$$
\frac{A(n-c)}{A(n)} \leqslant\left(\frac{2}{3}\right)^{\left(\begin{array}{c}
n \\
2
\end{array}\right)-\left(\begin{array}{c}
n-c \\
2
\end{array}\right)}=\left(\frac{2}{3}\right)^{c(2 n-c-1) / 2} .
$$

Proof. First of all, for $t \geqslant 1$ we have

$$
\frac{A(t-1)}{A(t)}=\frac{(2 t-1) !(2 t-2) !}{(3 t-2) !(t-1) !}=\frac{(2 t-2)(2 t-3) \cdots(t)}{(3 t-2)(3 t-3) \cdots(2 t)}
$$

and so

$$
\frac{A(t-1)}{A(t)} \leqslant\left(\frac{2(t-1)}{3(t-1)+1}\right)^{t-1} \leqslant\left(\frac{2}{3}\right)^{t-1} .
$$

But then

$$
\frac{A(n-c)}{A(n)}=\frac{A(n-c)}{A(n-c+1)} \cdot \frac{A(n-c+1)}{A(n-c+2)} \cdots \frac{A(n-1)}{A(n)},
$$

and the result follows from using (4) on each term.

Remark 5. The bound given in Lemma 4 can be significantly sharpened using either Stirling-type estimates or the asymptotic formula for $A(n)$, but we will only need this simple bound here.

Definition 6. Call row $i_{0}$ in a monotone triangle $\tau=(\tau(i, j)) \in \mathfrak{M}_{n}$ distinguished if it consists of the smallest possible entries $1,2,3, \ldots, i_{0}$, i.e.,

$$
\tau\left(i_{0}, j\right)=j \text { for } 1 \leqslant j \leqslant i_{0} .
$$

Notice that row $n$ is distinguished for any monotone triangle $\tau \in \mathfrak{M}_{n}$.

Notation 1. Let $\mathcal{D}(\tau)$ denote the set of distinguished rows for a given monotone triangle $\tau \in \mathfrak{M}_{n}$. Given a collection of row indices $\mathcal{I}=\left\{i_{1}, i_{2}, \ldots, i_{k}\right\} \subseteq[n-1]$, let $\eta_{n}(\mathcal{I})=$ $\eta_{n}\left(i_{1}, i_{2}, \ldots, i_{k}\right)$ denote the number of monotone triangles $\tau \in \mathfrak{M}_{n}$ such that $\mathcal{I} \cup\{n\} \subseteq$ $\mathcal{D}(\tau)$. When applicable, we will assume that $i_{1}<i_{2}<\cdots<i_{k}$. If no distinguished rows are specified outside of the (bottom) $n$-th row, we adopt the notation $\eta_{n}(\emptyset)$. That is, $\eta_{n}(\emptyset)$ is the number of monotone triangles $\tau \in \mathfrak{M}_{n}$ such that $\emptyset \cup\{n\} \subseteq \mathcal{D}(\tau)$; clearly we have $\eta_{n}(\emptyset)=A(n)$. 
Lemma 7. For any row indices $i_{1}, i_{2}, \ldots, i_{k} \in[n-1]$, we have

$$
\eta_{n}\left(i_{1}, i_{2}, \ldots, i_{k}\right)=A\left(i_{1}\right) A\left(i_{2}-i_{1}\right) \cdots A\left(i_{k}-i_{k-1}\right) A\left(n-i_{k}\right) .
$$

Proof. We first claim that

$$
\eta_{n}\left(i_{1}, i_{2}, \ldots, i_{k}\right)=A\left(i_{1}\right) \eta_{n-i_{1}}\left(i_{2}-i_{1}, \ldots, i_{k}-i_{1}\right)
$$

Indeed, if row $i_{1}$ is distinguished then the top $i_{1}$ rows form a monotone triangle of size $i_{1}$, hence the first factor of $A\left(i_{1}\right)$. The bottom $n-i_{1}$ rows must all start with the numbers $1,2,3, \ldots, i_{1}$ in their first $i_{1}$ entries; the remaining entries in these rows are in bijective correspondence with a monotone triangle of size $n-i_{1}$ given by subtracting $i_{1}$ from each of these entries, and row $i$ among the last $n-i_{1}$ rows in the original monotone triangle corresponds to row $i-i_{1}$ in this bijective element. As the rows $i_{2}, \ldots, i_{k}$, and $n$ are distinguished in the original monotone triangle, this means rows with transformed indices $i_{2}-i_{1}, \ldots, i_{k}-i_{1}$, and $n-i_{1}$ are distinguished in the corresponding monotone triangle of size $n-i_{1}$. Thus we get the second factor $\eta_{n-i_{1}}\left(i_{2}-i_{1}, \ldots, i_{k}-i_{1}\right)$.

The result now follows from (5) by induction on $k$.

Corollary 8. For any row indices $i_{1}, i_{2}, \ldots, i_{k} \in[n-1]$, we have

$$
\eta_{n}\left(i_{1}, i_{2}, \ldots, i_{k}\right) \leqslant A(n-k) .
$$

Proof. Using Lemmas 3 and 7, we have

$$
\begin{aligned}
\eta_{n}\left(i_{1}, i_{2}, \ldots, i_{k}\right) & =A\left(i_{1}\right) A\left(i_{2}-i_{1}\right) A\left(i_{3}-i_{2}\right) \cdots A\left(i_{k}-i_{k-1}\right) A\left(n-i_{k}\right) \\
& \leqslant A(1) A(1) A(1) \cdots A(1) A(n-k) .
\end{aligned}
$$

Remark 9. We should be careful to point out here that Corollary 8 will be of particular utility. It says that the number of monotone triangles containing a prescribed collection of $k$ distinguished rows (not including the very last $n$-th row) is at most

$$
\eta_{n}(1,2, \ldots, k)=\eta_{n}(n-k, n-k+1, \ldots, n-1)=A(n-k) .
$$

Said another way, the count of monotone triangles containing $k$ prescribed distinguished rows outside of row $n$ is no more than the count of monotone triangles with at least the top $k$ rows distinguished in addition to row $n$, or similarly the count of monotone triangle with at least the bottom $k+1$ rows distinguished (as both these counts are $A(n-k)$ by Lemma 7). 


\subsection{The proof}

By considering all $\left(\tau_{1}, \ldots, \tau_{r}\right)$ such that exactly one of them is $\tau_{\min }$, we have

$$
p_{\min } \geqslant \frac{r(A(n)-1)^{r-1}}{A(n)^{r}}=\frac{r(1-o(1))}{A(n)} .
$$

We will now produce a matching upper bound. Throughout the proof, we assume that $n$ is large enough to support our assertions.

Recall that row $i$ in a monotone triangle $\tau$ is distinguished if it consists of entries 1, 2, $\ldots, i$. Notice that in order for $\inf \left(\tau_{1}, \ldots, \tau_{r}\right)=\tau_{\text {min }}$, we must have each row distinguished in at least one of $\tau_{1}, \ldots, \tau_{r}$. This observation leads us to focus on the locations of the distinguished rows among the $\tau_{j}$.

For an $r$-tuple $\left(\tau_{1}, \ldots, \tau_{r}\right)$ with $\inf \left(\tau_{1}, \ldots, \tau_{r}\right)=\tau_{\min }$, let $D_{1}, \ldots, D_{r}$ be subsets of $[n]$ so that $D_{j}=\mathcal{D}\left(\tau_{j}\right)$ is the set of distinguished rows of $\tau_{j}$. Then the conditions

$$
D_{1} \cup \cdots \cup D_{r}=[n]
$$

and

$$
n \in D_{1} \cap \cdots \cap D_{n}
$$

must both hold. Using a trivial upper bound, there are at most $2^{r n}$ ways of choosing an $r$-tuple $\left(D_{1}, \ldots, D_{r}\right)$ satisfying $(7)$ and $(8)$.

Much of the rest of the proof is devoted to finding an upper bound on the number of $\left(\tau_{1}, \ldots, \tau_{r}\right)$ with $\inf \left(\tau_{1}, \ldots, \tau_{r}\right)=\tau_{\text {min }}$ that correspond to a particular $\left(D_{1}, \ldots, D_{r}\right)$. We will do this by producing an upper bound that holds for an entire class of $r$-tuples $\left(D_{1}, \ldots, D_{r}\right)$, and so we define these classes now.

For $i=0, \ldots, 6 r$, let $\mathcal{C}_{n-i}=\mathcal{C}_{n-i}(r)$ be the collection of all tuples $\left(\tau_{1}, \ldots, \tau_{r}\right)$ with $\inf \left(\tau_{1}, \ldots, \tau_{r}\right)=\tau_{\min }$ so that some $D_{j}$ (associated to $\tau_{j}$ ) has exactly $n-i$ consecutive elements from $[n]$, where $n-i$ is maximal in $D_{j}$ (and so, in particular, $D_{j}$ has no larger amount of consecutive elements from $[n])$. In other words, $\mathcal{C}_{n-i}$ consists of those $r$-tuples $\left(\tau_{1}, \ldots, \tau_{r}\right)$ with $\inf \left(\tau_{1}, \ldots, \tau_{r}\right)=\tau_{\min }$ so that some $\tau_{j}$ has a block of exactly $n-i$ consecutive distinguished rows. (It should be emphasized that whenever we say "consecutive" for the remainder of this paper, it should be interpreted as maximally consecutive as we have done here.) See Figure 2. Then let $\mathcal{C}_{\leqslant n-6 r-1}$ be the collection of all $\left(\tau_{1}, \ldots, \tau_{r}\right)$ with $\inf \left(\tau_{1}, \ldots, \tau_{r}\right)=\tau_{\min }$ so that all $D_{j}$ have at most $n-6 r-1$ consecutive elements from $[n]$. Clearly, for any $\left(\tau_{1}, \ldots, \tau_{r}\right)$ with $\inf \left(\tau_{1}, \ldots, \tau_{r}\right)=\tau_{\text {min }}$ we have $\left(\tau_{1}, \ldots, \tau_{r}\right) \in \mathcal{C}_{n} \cup \cdots \cup \mathcal{C}_{n-6 r} \cup \mathcal{C}_{\leqslant n-6 r-1}$.

First, notice that

$$
\left|\mathcal{C}_{n}\right| \leqslant r A(n)^{r-1},
$$

which follows since $\left(\tau_{1}, \ldots, \tau_{r}\right) \in \mathcal{C}_{n}$ implies that $D_{j}=[n]$ for some $j$. That is, some $\tau_{j}=\tau_{\min }$ in this case. Our goal is to show that the number of elements in the remaining classes is small relative to this upper bound.

Next, consider $\mathcal{C}_{n-1}$. How many $\left(\tau_{1}, \ldots, \tau_{r}\right)$ are in $\mathcal{C}_{n-1}$ ? There are $r$ choices for the monotone triangle $\tau_{j}$ which will have the $n-1$ consecutive distinguished rows. In fact, 


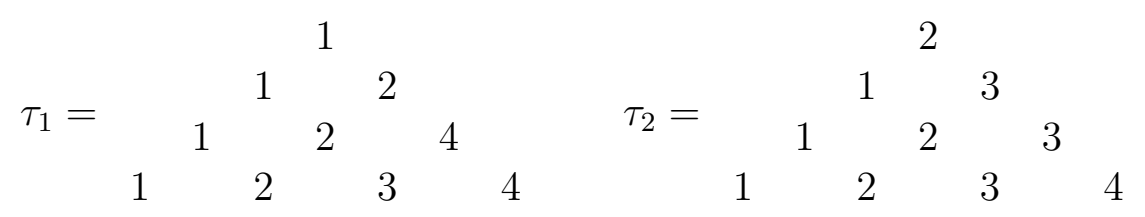

Figure 2: An example $\left(\tau_{1}, \tau_{2}\right)$ with $\inf \left(\tau_{1}, \tau_{2}\right)=\tau_{\min }$. In this example $D_{1}=\{1,2,4\}$, $D_{2}=\{3,4\}$, and $\left(\tau_{1}, \tau_{2}\right) \in \mathcal{C}_{4-2}$.

the $n-1$ consecutive distinguished rows must be rows $2,3, \ldots, n$, as $n \in D_{j}$, and so the first row of $\tau_{j}$ must be a 2 . There are $r-1$ choices for $k$ such that $\tau_{k}$ has a distinguished first row. Once $k$ is fixed, by Lemma 7 there are $A(n-1)$ possible monotone triangles $\tau_{k}$ satisfying this condition, and we place no restriction on the remaining $\tau_{\ell}$. This means that

$$
\left|\mathcal{C}_{n-1}\right| \leqslant r(r-1) A(n-1) A(n)^{r-2} .
$$

We'll consider $\mathcal{C}_{n-i}$ for $i=2, \ldots, 6 r$ via the following lemma.

Lemma 10. Let $r \geqslant 2$ and $i \in\{2,3, \ldots, 6 r\}$. Then

$$
\left|\mathcal{C}_{n-i}\right| \leqslant r(r-1)^{2} i A(i+1) A(i) A(n-i+1) A(n)^{r-2} .
$$

Proof. Choose (in $r$ ways) $j$ so that $\tau_{j}$ has exactly $n-i$ consecutive distinguished rows. There are $i$ places for the $n-i$ consecutive distinguished rows to occur (they can't be rows $i, \ldots, n-1)$. First assume that the block of $n-i$ rows is not the first or last $n-i$ rows; we will deal with these two cases later. Since there are $n-i$ distinguished rows in $[n-1]$, by Corollary 8 there are at most $A(i)$ possibilities for a monotone triangle $\tau_{j}$ that has a fixed placement of the $n-i$ consecutive distinguished rows.

Next, we choose $k \neq j$ corresponding to a monotone triangle $\tau_{k}$ that has a distinguished row directly preceding the $n-i$ consecutive distinguished rows of $\tau_{j}$, and similarly we choose $\ell \neq j$ corresponding to a monotone triangle $\tau_{\ell}$ (where potentially $\ell=k$ ) that has a distinguished row directly following the $n-i$ consecutive distinguished rows of $\tau_{j}$. Let $i_{1}$ be the number of undetermined rows that precede the $n-i$ distinguished rows of $\tau_{j}$, and let $i_{2}=i-i_{1}$. In other words, $\tau_{k}$ has row $i_{1}$ distinguished and $\tau_{\ell}$ has row $n-i_{2}+1$ distinguished.

If $k \neq \ell$, then by Lemma 7 there are $A\left(i_{1}\right) A\left(n-i_{1}\right) A\left(n-i_{2}+1\right) A\left(i_{2}-1\right)$ choices for monotone triangles $\tau_{k}$ and $\tau_{\ell}$ subject to these restrictions. As $i_{1}+i_{2}=i$ and $i_{1}, i_{2} \geqslant 1$, repeated applications of Lemma 3 delivers

$$
A\left(i_{1}\right) A\left(i_{2}-1\right) \leqslant A(i-1)
$$

and

$$
A\left(i_{1}\right) A\left(n-i_{1}\right) A\left(n-i_{2}+1\right) A\left(i_{2}-1\right) \leqslant A(i-1) A(n-i+1) A(n) .
$$

If $k=\ell$, then similarly we have at most $A(i-1) A(n-i+1)$ choices for $\tau_{k}$. Finally, we let the remaining $r-3(r-2$, if $k=\ell)$ monotone triangles be arbitrary. 
Putting these pieces together, in these $i-2$ cases we obtain the upper bound

$$
r(r-1)^{2} A(i) A(i-1) A(n-i+1) A(n)^{r-2} .
$$

Now what about the case where the block of $n-i$ distinguished rows is at the bottom of $\tau_{j}$ ? Here, we need only choose $k \neq j$ so that $\tau_{k}$ will have distinguished row $i$. Then, $\tau_{j}$ can be completed outside of its bottom $n-i$ distinguished rows in $A(i+1)$ ways, and $\tau_{k}$ can be completed outside of its distinguished $i$-th row in $A(i) A(n-i)$ ways. Letting the other $n-2$ monotone triangles be arbitrary delivers the bound

$$
r(r-1) A(i+1) A(i) A(n-i) A(n)^{r-2}
$$

in this case. A similar analysis shows that the case where the block of $n-i$ distinguished rows is at the top of $\tau_{j}$ delivers the bound

$$
r(r-1) A(i) A(i-1) A(n-i+1) A(n)^{r-2} .
$$

Combining the $i-2$ "intermediate" cases and their common bound given by (11) with the top/bottom cases and their bounds given by (12) and (13), we obtain

$$
\begin{aligned}
&\left|\mathcal{C}_{n-i}\right| \leqslant r(r-1)^{2}(i-2) A(i) A(i-1) A(n-i+1) A(n)^{r-2} \\
&+r(r-1) A(i+1) A(i) A(n-i) A(n)^{r-2} \\
&+r(r-1) A(i) A(i-1) A(n-i+1) A(n)^{r-2} \\
& \leqslant r(r-1)^{2} i A(i+1) A(i) A(n-i+1) A(n)^{r-2}
\end{aligned}
$$

as claimed.

Finally, we need to bound $\left|\mathcal{C}_{\leqslant n-6 r-1}\right|$. Here, we already know that there are at most $2^{r n}$ possible choices for an $r$-tuple $\left(D_{1}, \ldots, D_{r}\right)$ satisfying $(7)$ and (8). We fix such a $\left(D_{1}, \ldots, D_{r}\right)$ and bound the number of $r$-tuples $\left(\tau_{1}, \ldots, \tau_{r}\right) \in \mathcal{C}_{\leqslant n-6 r-1}$ that correspond to this fixed $r$-tuple $\left(D_{1}, \ldots, D_{r}\right)$.

A precise count of these $r$-tuples would be difficult to compute, but we only need an upper bound. But surely this count is at most

$$
\prod_{j=1}^{r} \eta_{n}\left(D_{j} \backslash\{n\}\right)
$$

indeed, the number of $\tau \in \mathfrak{M}_{n}$ with precisely the distinguished rows $D_{j}$ is certainly at most the number of $\tau \in \mathfrak{M}_{n}$ such that $D_{j} \subseteq \mathcal{D}(\tau)$. Then, from Corollary 8 we have

$$
\prod_{j=1}^{r} \eta_{n}\left(D_{j} \backslash\{n\}\right) \leqslant \prod_{j=1}^{r} A\left(n-\left|D_{j}\right|+1\right) .
$$

Now let index $m$ be such that $\left|D_{m}\right|$ is maximal. First, we'll suppose that $\left|D_{m}\right| \leqslant n-6 r$. Notice that

$$
\left(\left|D_{1}\right|-1\right)+\left(\left|D_{2}\right|-1\right)+\cdots+\left(\left|D_{r}\right|-1\right) \geqslant n-1
$$


which implies that

$$
\left|D_{m}\right| \geqslant \frac{n-1}{r}+1=\frac{n+r-1}{r} \geqslant \frac{n}{r}
$$

and so

$$
\left|D_{m}\right| \geqslant\lceil n / r\rceil
$$

Then by Lemma 3 we have

$$
\prod_{j=1}^{r} A\left(n-\left|D_{j}\right|+1\right) \leqslant A\left(n-\left|D_{m}\right|+1\right) A(\delta) A(n)^{r-2},
$$

where $\delta:=\max \left\{n-\left|D_{1}\right|-\left|D_{2}\right|-\cdots-\left|D_{r}\right|+r+\left(\left|D_{m}\right|-1\right), 0\right\}$. Indeed, let $m_{2} \neq m$ denote the index of the second largest value among $\left|D_{1}\right|, \ldots,\left|D_{n}\right|$. All we have done in order to obtain (16) is to leave the $m$-th factor alone while repeatedly increasing the argument sizes of the $r-2$ factors with index not in $\left\{m, m_{2}\right\}$, one at a time, always at the expense of the factor with index $m_{2}$ (via Lemma 3 ). If it should happen that factor $m_{2}$ 's argument decreases to 0 in the process, we then leave that argument alone and simply continue to increase the other $r-2$ arguments (other than the $m$-th) all the way up to $n$.

Then using $\left|D_{m}\right| \leqslant n-6 r,(15)$, and (16), we see that

$$
\begin{aligned}
\prod_{j=1}^{r} A\left(n-\left|D_{j}\right|+1\right) & \leqslant A\left(n-\left|D_{m}\right|+1\right) A(\delta) A(n)^{r-2} \\
& \leqslant A\left(n-\left|D_{m}\right|+1\right) A\left(\left|D_{m}\right|\right) A(n)^{r-2} \\
& \leqslant A(6 r+1) A(n-6 r) A(n)^{r-2},
\end{aligned}
$$

where the last inequality uses Lemma 3 along with $\lceil n / r\rceil \leqslant\left|D_{m}\right| \leqslant n-6 r$ (and so $A(n-\lceil n / r\rceil+1) A(\lceil n / r\rceil) \leqslant A(6 r+1) A(n-6 r)$ for large enough $n)$.

If instead $\left|D_{m}\right|>n-6 r$, then we consider the $n-6 r$ consecutive rows $3 r, 3 r+1, \ldots, n-$ $3 r-1$. Since $\tau_{m}$ cannot have $n-6 r$ consecutive distinguished rows, by (7) we know that some other $\tau_{k}$ must have a distinguished row from among rows $3 r, 3 r+1, \ldots, n-3 r-1$. Corollary 8 gives at most $A\left(n-\left|D_{m}\right|+1\right) \leqslant A(6 r)$ choices for such a $\tau_{m}$, and Lemmas 7 and 3 give at most $A(3 r) A(n-3 r)$ choices for such a $\tau_{k}$. Putting no restrictions on the remaining monotone triangles, we have at most

$$
A(6 r) A(3 r) A(n-3 r) A(n)^{r-2}
$$

monotone triangles corresponding to $\left(D_{1}, \ldots, D_{r}\right)$ where $\left|D_{m}\right|>n-6 r$. Since there are at most $2^{r n} r$-tuples $\left(D_{1}, \ldots, D_{r}\right)$ and from (17) and (18) each of these corresponds to at most $A(6 r) A(3 r) A(n-3 r) A(n)^{r-2} r$-tuples $\left(\tau_{1}, \ldots, \tau_{r}\right)$ with $\inf \left(\tau_{1}, \ldots, \tau_{r}\right)=\tau_{\min }$, for large enough $n$ we have

$$
\left|\mathcal{C}_{\leqslant n-6 r-1}\right| \leqslant 2^{r n} A(6 r) A(3 r) A(n-3 r) A(n)^{r-2} .
$$


We are now ready to finish the calculation. We have (with the main inequalities justified below, and all implied constants depending on $r$ )

$$
\begin{aligned}
p_{\min } & =\frac{\left|\left\{\left(\tau_{1}, \ldots, \tau_{r}\right): \inf \left(\tau_{1}, \ldots, \tau_{r}\right)=\tau_{\min }\right\}\right|}{A(n)^{r}} \\
& \leqslant \frac{\left|\mathcal{C}_{n}\right|+\left|\mathcal{C}_{n-1}\right|+\cdots+\left|\mathcal{C}_{n-6 r}\right|+\left|\mathcal{C}_{\leqslant n-6 r-1}\right|}{A(n)^{r}} \\
& \leqslant \frac{r A(n)^{r-1}+O\left(A(n-1) A(n)^{r-2}\right)+O\left(2^{r n} A(n-3 r) A(n)^{r-2}\right)}{A(n)^{r}} \\
& \leqslant \frac{r+O\left((2 / 3)^{n}\right)+O\left(2^{r n}(2 / 3)^{3 r n}\right)}{A(n)} \\
& =\frac{r(1+o(1))}{A(n)},
\end{aligned}
$$

where (20) follows from (9), (10), Lemma 3, Lemma 10 and (19), while (21) follows from Lemma 4. Therefore, from (6) and (22) we have

$$
p_{\min } \sim \frac{r}{A(n)}
$$

\section{Second-order term}

In this section, we describe how the ideas from the proof of Theorem 1 can be generalized to produce a second-order term for $p_{\min }$. By inspection, there are 1, 1, and 6 monotone triangles with a maximal block of exactly $n, n-1$, and $n-2$ consecutive distinguished rows, respectively. In particular,

there are $A(n)-8$ monotone triangles of size $n$

with at most $n-3$ consecutive distinguished rows.

Let $\tau_{\min }^{\prime}$ denote the monotone triangle obtained from $\tau_{\min }$ by changing the top row to a 2 , and $\tau_{\min }^{\prime \prime}$ denote the monotone triangle obtained from $\tau_{\min }$ by changing row $n-1$ to $1,2,3, \cdots, n-3, n-2, n$. It is important to note that $\tau_{\min }^{\prime}$ and $\tau_{\min }^{\prime \prime}$ each have more than $n-3$ consecutive distinguished rows.

We produce a lower bound as follows: Consider all $\left(\tau_{1}, \ldots, \tau_{r}\right)$ such that:

1. one $\tau_{j}$ is $\tau_{\min }$, and the others have at most $n-3$ consecutive distinguished rows; or

2. one $\tau_{j}$ is $\tau_{\min }^{\prime}$, a second $\tau_{k}$ has row 1 distinguished and has at most $n-3$ consecutive distinguished rows, and the remaning $\tau_{\ell}$ do not have row 1 distinguished and have at most $n-3$ consecutive distinguished rows; or

3. one $\tau_{j}$ is $\tau_{\min }^{\prime \prime}$, a second $\tau_{k}$ has row $n-1$ distinguished and has at most $n-3$ consecutive distinguished rows, and the remaining $\tau_{\ell}$ do not have row $n-1$ distinguished and have at most $n-3$ consecutive distinguished rows. 
Notice that the collections of $r$-tuples in the three cases above are disjoint, since exactly one monotone triangle $\tau_{j}$ will be either $\tau_{\min }, \tau_{\min }^{\prime}$, or $\tau_{\min }^{\prime \prime}$. Using $(23)$, there are $r(A(n)-$ $8)^{r-1}=r A(n)^{r-1}-\Theta\left(A(n)^{r-2}\right) r$-tuples $\left(\tau_{1}, \ldots, \tau_{r}\right)$ in the first collection.

For the second collection, there are $r$ choices for the index $j$ followed by $r-1$ choices for $k$. To compute the number of possibilities for $\tau_{k}$ in this collection we use a union bound: subtract the total number of monotone triangles that do not have row 1 distinguished (there are $A(n)-A(n-1)$ of these) from the number of monotone triangles with at most $n-3$ consecutive distinguished rows given by $(23)$ to obtain at least $(A(n)-8)-(A(n)-$ $A(n-1))=A(n-1)-8$ choices for $\tau_{k}$. Lastly, from (23) and another union bound there are at least $(A(n)-8)-A(n-1)$ choices for each of the $r-2$ remaining monotone triangles $\tau_{\ell}$.

The enumeration for the third collection is very similar. There are $r$ choices for $j$, $r-1$ choices for $k$, at least $A(n-1)-8$ choices for $\tau_{k}$, and at least $A(n)-A(n-1)-8$ choices for each of the $r-2$ remaining monotone triangles $\tau_{\ell}$. Therefore,

$$
\begin{aligned}
p_{\min } \geqslant & \frac{r(A(n)-8)^{r-1}+r(r-1)(A(n-1)-8)(A(n)-A(n-1)-8)^{r-2}}{A(n)^{r}} \\
& +\frac{r(r-1)(A(n-1)-8)(A(n)-A(n-1)-8)^{r-2}}{A(n)^{r}} \\
= & \frac{r A(n)^{r-1}+2 r(r-1) A(n-1) A(n)^{r-2}-O\left(A(n-1)^{2} A(n)^{r-3}\right)}{A(n)^{r}}
\end{aligned}
$$

For a corresponding upper bound, we use

$$
\left|\mathcal{C}_{n}\right| \leqslant r A(n)^{r-1} \text { and }\left|\mathcal{C}_{n-1}\right| \leqslant r(r-1) A(n-1) A(n)^{r-2},
$$

as before. We can refine $\mathcal{C}_{n-2}$ to find those $r$-tuples with a $\tau_{j}$ having the top $n-2$ rows distinguished (giving 1 choice for the last entry in row $n-1$ of $\tau_{j}$, as row $n-1$ is not distinguished; i.e., $\tau_{j}=\tau_{\min }^{\prime \prime}$ ), and a $k \neq j$ so that $\tau_{k}$ has row $n-1$ distinguished. There are $r$ choices for $j, r-1$ choices for $k, A(n-1)$ choices for $\tau_{k}$, and we let the rest of the choices be arbitrary. This gives an upper bound of $r(r-1) A(n-1) A(n)^{r-2}$ for the size of this subset of $\mathcal{C}_{n-2}$. From the proof of Lemma 10 the remaining subset of $\mathcal{C}_{n-2}$ has size at most $O\left(A(n-2) A(n)^{r-2}\right)$ (specifically, see the discussion around (12)).

Using the bounds for $\left|\mathcal{C}_{n-3}\right|, \ldots,\left|\mathcal{C}_{n-6 r}\right|$ obtained in Lemma 10 and the bound for $\left|\mathcal{C}_{\leqslant n-6 r-1}\right|$ from (19), we have

$$
\begin{aligned}
p_{\min } \leqslant & \frac{r A(n)^{r-1}+2 r(r-1) A(n-1) A(n)^{r-2}}{A(n)^{r}} \\
& +\frac{O\left(A(n-2) A(n)^{r-2}\right)+O\left(2^{r n} A(n-3 r) A(n)^{r-2}\right)}{A(n)^{r}} \\
= & \frac{r A(n)^{r-1}+2 r(r-1) A(n-1) A(n)^{r-2}}{A(n)^{r}}
\end{aligned}
$$




$$
\begin{array}{r}
+\frac{O\left(A(n-2) A(n)^{r-2}\right)+O\left(2^{r n} A(n-2)(2 / 3)^{(3 r-2) n} A(n)^{r-2}\right)}{A(n)^{r}} \\
=\frac{r A(n)^{r-1}+2 r(r-1) A(n-1) A(n)^{r-2}+O\left(A(n-2) A(n)^{r-2}\right)}{A(n)^{r}}
\end{array}
$$

here, moving from line (25) to (26) we have used $r \geqslant 2$.

Notice that $A(n-1)^{2} \leqslant A(n-2) A(n)$ (via Lemma 3), and so (24) and (26) imply that

$$
p_{\text {min }}=\frac{r A(n)^{r-1}+2 r(r-1) A(n-1) A(n)^{r-2}+\Theta\left(A(n-2) A(n)^{r-2}\right)}{A(n)^{r}} .
$$

\section{References}

[1] P. Bleher and V. Fokin, Exact Solution of the Six-Vertex Model with Domain Wall Boundary Conditions. Disordered Phase, Commun. Math. Phys. 268 (2006), 223284.

[2] D. M. Bressoud, Proofs and Confirmations: The Story of the Alternating Sign Matrix Conjecture, MAA, Cambridge University Press, Cambridge, 1999.

[3] D. M. Bressoud and J. Propp, How the alternating sign matrix conjecture was solved, Notices of the AMS 46(6) (1999), 637-646.

[4] E. R. Canfield, Meet and join within the lattice of set partitions, Electronic J. Combinatorics 8(1) (2001), \#R15.

[5] W. Y. C. Chen and D. G. L. Wang, Minimally intersecting set partitions of type $B$, Electronic J. Combinatorics 17 (2010), \#R22.

[6] C. Ehresmann, Sur la topologie de certains espaces homogénes, Ann. Math. 35 (1934), 396-443.

[7] I. Fischer, The number of monotone triangles with prescribed bottom row, $A d v$. in Appl. Math. 37(2) (2006), 249-267.

[8] I. Fischer, The operator formula for monotone triangles - simplified proof and three generalizations, J. Combin. Theory Ser. A 117 (2010), 1143-1157.

[9] I. Fischer, Refined enumerations of alternating sign matrices: monotone $(d, m)$ trapezoids with prescribed top and bottom row, J. Algebr. Comb. 33 (2010), 239257.

[10] A. Hammett, On comparibility of random permutations, Ph. D. Thesis, The Ohio State University, 2007.

[11] A. Hammett and B. Pittel, How often are two permutations comparable? Trans. of the AMS 360(9) (2008), 4541-4568.

[12] G. Kuperberg, Another proof of the alternating sign matrix conjecture, Int. Math. Res. Not. 3 (1996), 139-150.

[13] A. Lascoux and M. P. Schützenberger, Treillis et bases des groupes de Coxeter, Electronic J. Combinatorics 3 (1996), \#R27. 
[14] B. Pittel, Where the typical set partitions meet and join, Electronic J. Combinatorics 7 (2000), \#R5.

[15] J. Propp, The many faces of alternating-sign matrices, Discrete Mathematics and Theoretical Computer Science Proceedings AA (DM-CCG) (2001), 43-58.

[16] R. P. Stanley, Enumerative Combinatorics, Volume 2, Cambridge University Press, Cambridge, 1999.

[17] J. Striker, A direct bijection between descending plane partitions with no special parts and permutation matrices, Discrete Math. 311 (2011), 2581-2585.

[18] D. Zeilberger, Proof of the alternating sign matrix conjecture, Electronic J. Combinatorics 3(2) (1996), \#R13. 